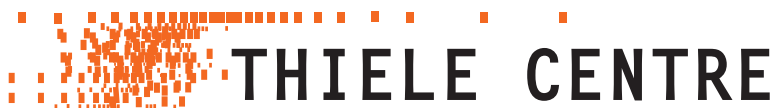 FOR APPLIED MATHEMATICS IN NATURAL SCIENCE
}

Self-scaling of Turbulent Energy Dissipation Correlators

\section{Jürgen Schmiegel}




\section{Self-scaling of Turbulent Energy Dissipation Correlators}

This Thiele Research Report is also Research Report number 448 in the Stochastics Series at Department of Mathematical Sciences, University of Aarhus, Denmark. 



\title{
Self-scaling of turbulent energy dissipation correlators
}

\author{
Jürgen Schmiegel \\ Department of Mathematical Sciences, \\ University of Aarhus \\ DK-8000 Aarhus, Denmark *
}

\begin{abstract}
In a number of recent papers $[1,2,3,4]$, a continuous spatiotemporal process based on the integration of Lévy bases was proposed to describe the statistics of the turbulent energy dissipation. An immediate consequence of this model is self-scaling of dissipation correlators, similar to the concept of Extended Self Similarity (ESS) for velocity increments. We report empirical findings about self-scaling of dissipation correlators that support the Lévy based dynamical modeling of the energy dissipation.
\end{abstract}

PACS: 47.27.-i

KEYWORDS: Turbulence, Lévy basis, correlations, scaling.

*The author is much indebted to K.R. Sreenivasan, J. Peinke, B.R. Pearson and B. Chabaud for allowing us to use the data sets. J.S. acknowledges support from the Carlsberg Foundation. 


\section{Introduction}

Geometric cascade processes play a fundamental role for modeling strongly anomalous, intermittent fluctuations, long-range correlations, multi-scale structuring and self-similarity. In particular, they have been proved and tested in describing stylized facts of the statistics of the energy-dissipation in turbulence $[5,6,7,8]$.

Various generalisations of such purely spatial and discrete processes towards continuous cascade processes in time and/or space, formulated in terms of integrals over an uncorrelated noise field, have been undertaken recently $[1,2,3,4,9,10]$. A spatio-temporal generalisation in a continuous framework that is causal is proposed in [4]. It was shown that this process can be constructed in analogy to a discrete cascade process and analytical forms for twoand three-point correlations were successfully compared to the corresponding experimental statistics in fully developed turbulent shear flow. In particular, it was demonstrated that two- and three-point correlations follow a simple scaling relation for a certain, but extended range of scales. As a generalisation of these results, [2] proved that scaling n-point correlations imply scaling relations for the moments of the coarse grained energy-dissipation which is usually associated to the multifractal structure of the energy-dissipation field.

However, scaling of moments of the coarse grained energy-dissipation and n-point correlations is not perfect. Pure scaling relations are expected only within the so called inertial subrange for very high Reynolds numbers. The present paper investigates the form of two-point correlations of the energydissipation field for various data sets, in particular for low and moderate Reynolds numbers. It turns out that two-point correlations of the energydissipation can be expressed in analogy to the concept of Extended SelfSimilarity [11] for the turbulent velocity field. Furthermore, we show that this kind of self-scaling follows immediately within the continuous framework proposed in $[1,2,3,4]$.

Section 2 reviews the definition and basic properties of the Lévy based model. Section 3 provides a detailed analysis of various data sets that supports the concept of self-scaling of two-point correlators. Section 4 discusses the empirical results and Section 5 concludes.

\section{Lévy based model}

This Section briefly describes a class of causal spatio-temporal processes that are based on the integration of an independently scattered random measure of Lévy type. These processes comprise, as special cases, the temporal cascade 
processes discussed in [9] and [10], as well as the spatio-temporal cascade process derived in [4].

The basic notion is that of an independently scattered random measure (i.s.r.m) on continous space-time, $\mathbb{R} \times \mathbb{R}$. These measures associate a random number with any subset of $\mathbb{R} \times \mathbb{R}$. Whenever two subsets are disjoint, the associated numbers are independent, and the measure of a disjoint union of sets almost certainly equals the sum of the measures of the individual sets. For a mathematically more rigorous definition of i.s.r.m.'s and their theory of integration, see $[3,12,13]$.

Independently scattered random measures provide a natural basis for describing uncorrelated noise processes in space and time. A special class of i.s.r.m.'s is that of factorisable and homogeneous Lévy bases, where the distribution of the measure of each set is infinitely divisible and does not depend on the location of the subset. In this case, it is easy to handle integrals with respect to the Lévy basis using the well-known Lévy-Khintchine and LévyIto representations for Lévy processes. Here, we state the result and point to [3] for greater detail and rigour.

Let $Z$ be a factorisable and homogeneous Lévy basis on $\mathbb{R} \times \mathbb{R}$, i.e. $Z(A)$ is infinitely divisible for any $A \subset \mathbb{R} \times \mathbb{R}$. Then we have the fundamental relation

$$
\left\langle\exp \left\{\int_{A} h(a) Z(\mathrm{~d} a)\right\}\right\rangle=\exp \left\{\int_{A} \mathrm{~K}[h(a)] \mathrm{d} a\right\},
$$

where $\langle\cdots\rangle$ denotes the expectation, $h$ is any integrable deterministic function, and $\mathrm{K}$ denotes the cumulant function of $Z(\mathrm{~d} a)$, defined by

$$
\ln \langle\exp \{\xi Z(\mathrm{~d} a)\}\rangle=\mathrm{K}[\xi] \mathrm{d} a .
$$

The usefulness of (1) is obvious: it permits explicit calculation of the correlation function of the integrated and $h$-weighted noise field $Z(\mathrm{~d} a)$ once the cumulant function $\mathrm{K}$ of $h$ is known.

Based on relation (1), we construct a spatio-temporal process for the $(1+$ 1) dimensional energy dissipation in turbulence that is causal and continuous [14] by defining the energy dissipation field $\epsilon(x, t)$ as

$$
\epsilon(x, t)=\exp \left\{\int_{A_{t}(x)} Z\left(\mathrm{~d} x^{\prime} \times \mathrm{d} t^{\prime}\right)\right\},
$$

where we have set $h(a) \equiv 1$. (3) constitutes a multiplicative process of independent factors $\exp \left\{Z\left(\mathrm{~d} x^{\prime} \times \mathrm{d} t^{\prime}\right)\right\}$ made up of a factorisable and homogeneous Lévy basis $Z$ over $\mathbb{R} \times \mathbb{R}$. Contributions to field amplitude $\epsilon(x, t)$ lie within the influence domain $A_{t}(x)$, called the associated ambit set. 


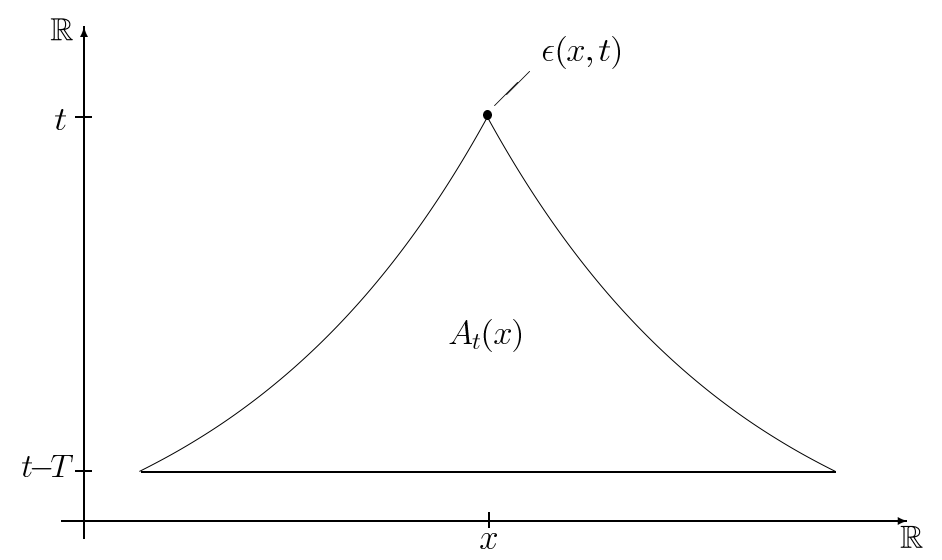

Figure 1: Illustration of the ambit set $A_{t}(x)$ associated to $\epsilon(x, t)$.

Figure 1 illustrates the construction of the energy dissipation field. The decorrelation time $T$ denotes the temporal extension of the ambit set in the past.

The generality of the model (3) is based on the possibility of choosing the constituents of the process $\epsilon(x, t)$ independently. The available degrees of freedom are an arbitrary infinitely divisible distribution for the Lévy basis $Z$ (including Brownian motion, stable processes, self-decomposable processes etc.) and the shape of the ambit set $A$.

Despite its generality, the model is tractable enough to yield explicit expressions for arbitrary $n$-point correlations $\left\langle\epsilon\left(x_{1}, t_{1}\right) \cdot \ldots \epsilon\left(x_{n}, t_{n}\right)\right\rangle$ in closed form. In this paper, the focus is on two-point correlators of order $\left(n_{1}, n_{2}\right)$, defined as

$$
c_{n_{1}, n_{2}}\left(x_{1}, t_{1} ; x_{2}, t_{2}\right) \equiv \frac{\left\langle\epsilon\left(x_{1}, t_{1}\right)^{n_{1}} \epsilon\left(x_{2}, t_{2}\right)^{n_{2}}\right\rangle}{\left\langle\epsilon\left(x_{1}, t_{1}\right)^{n_{1}}\right\rangle\left\langle\epsilon\left(x_{2}, t_{2}\right)^{n_{2}}\right\rangle} .
$$

Using (1), it is straightforward to show $[2,3,4]$

$$
c_{n_{1}, n_{2}}\left(x_{1}, t_{1} ; x_{2}, t_{2}\right)=\exp \left\{\overline{\mathrm{K}}\left[n_{1}, n_{2}\right] \int_{A_{t_{1}\left(x_{1}\right) \cap A_{t_{2}}\left(x_{2}\right)}} \mathrm{d} x \mathrm{~d} t\right\},
$$

with the abbreviation $\overline{\mathrm{K}}\left[n_{1}, n_{2}\right]=\mathrm{K}\left[n_{1}+n_{2}\right]-\mathrm{K}\left[n_{1}\right]-\mathrm{K}\left[n_{2}\right]$. The important point here is the fact that the exponent in (5) factorizes into the overlap of the two ambit sets times a factor depending only on the order $\left(n_{1}, n_{2}\right)$. Thus we are able to rewrite (5) as a self-scaling relation of two point correlators of orders $\left(n_{1}, n_{2}\right)$ and $\left(m_{1}, m_{2}\right)$

$$
c_{n_{1}, n_{2}}\left(x_{1}, t_{1} ; x_{2}, t_{2}\right)=c_{m_{1}, m_{2}}\left(x_{1}, t_{1} ; x_{2}, t_{2}\right)^{k\left[m_{1}, m_{2} ; n_{1}, n_{2}\right]}
$$


with the abbreviation

$$
k\left[m_{1}, m_{2} ; n_{1}, n_{2}\right]=\frac{\overline{\mathrm{K}}\left[n_{1}, n_{2}\right]}{\overline{\mathrm{K}}\left[m_{1}, m_{2}\right]},
$$

called the self-scaling exponent. This is the fundamental implication of the Lévy based model (3) we adress in this paper. We provide empirical evidence for (6) by analyzing a number of different turbulent data sets, with Taylor Reynolds numbers ranging from 80 up to 17000.

It is to note that (6) is similar to the concept of Extended Self-Similarity (ESS) [11] for the statistics of the turbulent velocity field. ESS claims that moments of velocity increments $u(s)$ of order $n$ show a pronounced scaling behaviour over a certain range of separations $s$ when plotted as a function of the moments of some different order $m$

$$
\left\langle u(s)^{n}\right\rangle=A_{n, m}\left\langle u(s)^{m}\right\rangle^{\tau(n, m)}
$$

with constant factors $A_{n, m}$ and constant, apparently universal exponents $\tau(n, m)$. A fundamental conceptual difference between (6) and (8) lies in the fact that (6) is expected to hold for arbitrary orders $\left(n_{1}, n_{2}\right)$ and $\left(m_{1}, m_{2}\right)$, including the case $m_{1} / n_{1} \neq m_{2} / n_{2}$. The later case means that $\epsilon\left(x_{1}, t_{1}\right)^{m_{1}} \epsilon\left(x_{2}, t_{2}\right)^{m_{2}}$ is not a power of $\epsilon\left(x_{1}, t_{1}\right)^{n_{1}} \epsilon\left(x_{2}, t_{2}\right)^{n_{2}}$. In contrast to that, (8) connects the statistics of $u(s)^{n}$ with that of its power $\left(u(s)^{n}\right)^{m / n}$. Moreover, the ESS-relation (8) contains adjustable parameters $A_{n, m}$, while for the self-scaling relation (6) the constant of proportionality is equal to one for all orders $\left(n_{1}, n_{2}\right)$ and $\left(m_{1}, m_{2}\right)$.

\section{Self-scaling of correlators}

The data sets we analysed in view of the self-scaling relation (6) consist of one-point time records of the longitudinal (along the mean flow) velocity component. The data are from the atmospheric boundary layer (data set $(\mathrm{A})$ ) $[15,16]$, from a gaseous helium jet flow (data sets (H1)-(H4)) [17], from a free air jet experiment (data set $(F)$ ) [18] and from a wind tunnel experiment (data set $(\mathrm{W})$ ) [19]. This collection of data sets comprise a wide range of Reynolds numbers from 80 (W) up to 17000 (A). Table 1 lists the Taylor Reynolds numbers $R_{\lambda}$ and the integral length scales $L$ (in units of the finest resolution) obtained as the integral over the velocity autocorrelation function (using Taylor's hypothesis). As is the standard practice, we invoke Taylor's hypothesis and calculate the one-dimensional surrogate energy-dissipation

$$
\epsilon(x)=15 \nu\left(\frac{\mathrm{d} u}{\mathrm{~d} x}\right)^{2} .
$$


Table 1: Taylor Reynolds numbers $R_{\lambda}$ and the estimated integral length scales $L$ (in units of the finest resolution) for the seven data sets.

\begin{tabular}{l|ccccccc}
\hline data set & $(\mathrm{A})$ & $(\mathrm{W})$ & $(\mathrm{F})$ & $(\mathrm{H} 1)$ & $(\mathrm{H} 2)$ & $(\mathrm{H} 3)$ & $(\mathrm{H} 4)$ \\
$R_{\lambda}$ & 17000 & 80 & 190 & 85 & 124 & 208 & 352 \\
$L$ & 20000 & 50 & 240 & 85 & 168 & 196 & 270 \\
\hline
\end{tabular}

Note that this construction gives the energy-dissipation at a fixed time, thus we omit the time coordinate of $\epsilon(x, t)$ in the following. We normalize the energy dissipation field $\langle\epsilon(x)\rangle=1$, implying $\mathrm{K}[1]=0$ and $\overline{\mathrm{K}}[2]=\mathrm{K}[2]$.

We perform our analysis of (6) for all combinations of the orders $(1,1)$, $(1,2)$, and $(2,2)$. Although we believe that our results also hold for higher order statistics, the quality of the data does not alllow to investigate higher order correlators with confidence. Note that $(2,2)$ corresponds to velocity statistics of order 8 .

Figure 2 shows the correlators $c_{n_{1}, n_{2}}(s)$ of data set (H3) as a function of the lag $s$ in double logarithmic representation. Here and in subsequent Figures $s$ is measured in units of the finest resolution. For the very large lags $s \in[50,200]$ it seems to be feasible to fit a straight line to all three graphs, corresponding to a scaling relation

$$
c_{n_{1}, n_{2}}(s)=B_{n_{1}, n_{2}} s^{-\xi\left(n_{1}, n_{2}\right)}
$$

with constant factors $B_{n_{1}, n_{2}}$ and constant exponents $\xi\left(n_{1}, n_{2}\right)$. Of particular interest is the case $\left(n_{1}, n_{2}\right)=(1,1)$, defining the intermittency exponent $\mu=\xi(1,1)[21,22,23]$. For the very small lags $s$ we observe a strong increase of correlations which is associated to surrogacy effects [20]. Due to the approximate scaling of $c_{n_{1}, n_{2}}(s)$ at large lags, we expect the self-scaling relation (6) to hold for lags $s \in[50,200]$.

Figure 3 shows the two-point correlator $c_{n_{1}, n_{2}}(s)$ as function of $c_{m_{1}, m_{2}}(s)$ in double logarithmic representation, again for data set (H3). It clearly confirms the self-scaling relation (6) with high accuracy for nearly all values of $c_{m_{1}, m_{2}}$ that are displayed in Figure 2, extending the scaling range $[50,200]$ (Figure 2) to $[5,200]$ (Figure 3). For the not too small lags $s$ constant slopes $k\left[m_{1}, m_{2} ; n_{1}, n_{2}\right]$ are well defined. Since $k[2,2 ; 1,2]=k[2,2 ; 1,1] / k[1,2 ; 1,1]$, the self-scaling behaviour in Figure $3(\mathrm{c})$ is an immediate consequence of the self-scaling behaviour in Figures 3(a) and 3(b).

Note that a scaling relation for $c_{n_{1}, n_{2}}(s)$, as observed for large lags in Figure 2 does not necessarily imply the self-scaling relation(6). In this case the validity of (6) additionally requires

$$
B_{n_{1}, n_{2}}^{1 / \xi\left(n_{1}, n_{2}\right)}=B_{m_{1}, m_{2}}^{1 / \xi\left(m_{1}, m_{2}\right)} .
$$


Figures 4, 5 show the corresponding results for data set (F). Again, we confirm the self-scaling relation (6) for nearly all lags (Figure 5). The scaling of $c_{n_{1}, n_{2}}(s)$ as a function of the lag $s$ is again restricted to the very large lags (Figure 4).

Similar results hold for the other data sets (H1), (H2), (H4) and (W). For data set (A) correlators show more scatter compared to the other data sets (Figure 6). For this high Reynolds number data set we expect the scaling relation $c_{1,1}(s) \propto s^{-\mu}$ [21] for a wide range of lags $s$ which is clearly displayed in Figure 6. For the higher orders, scatter increases. However, the absence of any trend makes it reasonable to assume (10) for $\left(n_{1}, n_{2}\right)=(1,2)$ and $\left(n_{1}, n_{2}\right)=(2,2)$ and all lags $s$. In this case the self-scaling relation (6) reduces to the condition (11). Figure 7 shows $c_{n_{1}, n_{2}}(s)$ as function of $c_{m_{1}, m_{2}}(s)$ and clearly supports the self-scaling relation (6).

In summary, we showed that the self-scaling relation (6) holds for all Reynolds numbers and nearly all lags $s$. For the very large Reynolds number it is related to scaling of correlators as a function of the lag $s$ under the condition (11). For the small and moderate Reynolds numbers and not too large lags $s$ self-scaling (6) is not related to scaling of correlators as a function of the lag $s$. The corresponding exponents $k\left[m_{1}, m_{2} ; n_{1}, n_{2}\right]$ can be estimated with high accuracy for all Reynolds numbers. These exponents are shown in Figure 8 for $n_{1}, n_{2}, m_{1}, m_{2} \in[1,2]$ as a function of the Reynolds number. Paying attention to the scatter, we are not able to clearly detect any Reynolds number dependence. A further clarification of this points requires investigation of higher order correlators. For the moment, we conclude that a possible Reynolds number dependence is very weak and does not show for the low orders. This is a remarkable result and similar to ESS where the reduced scaling exponents $\tau\left(n_{1}, n_{2}\right)$ (8) also seem to be Reynolds number independent. 

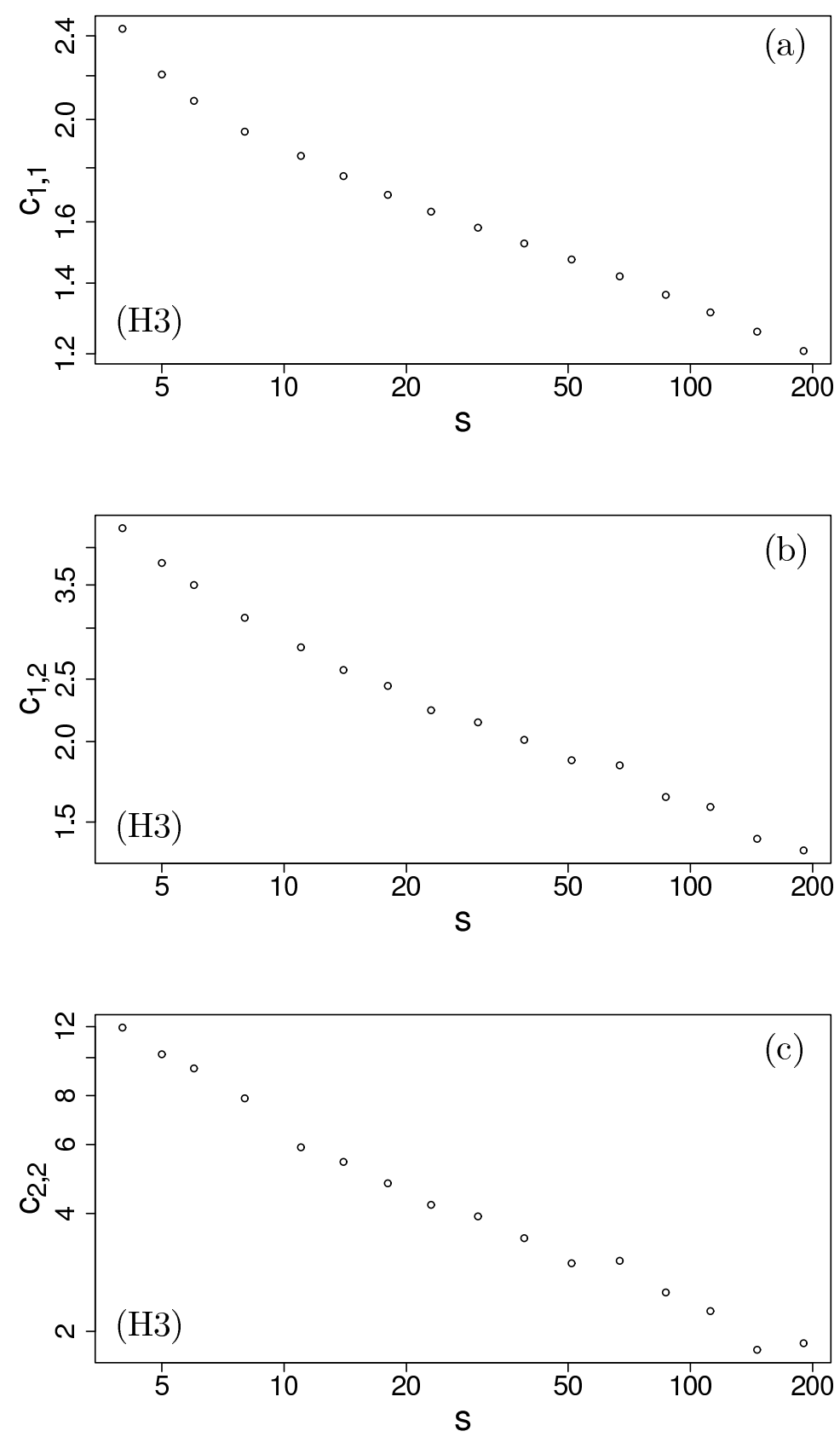

Figure 2: Energy dissipation correlators $c_{n_{1}, n_{2}}$ as a function of the lag $s$ (in units of the finest resolution) for data set (H3) and orders (a) $\left(n_{1}, n_{2}\right)=$ $(1,1)$, (b) $\left(n_{1}, n_{2}\right)=(1,2)$ and $(\mathrm{c})\left(n_{1}, n_{2}\right)=(2,2)$ in double logarithmic representation. 

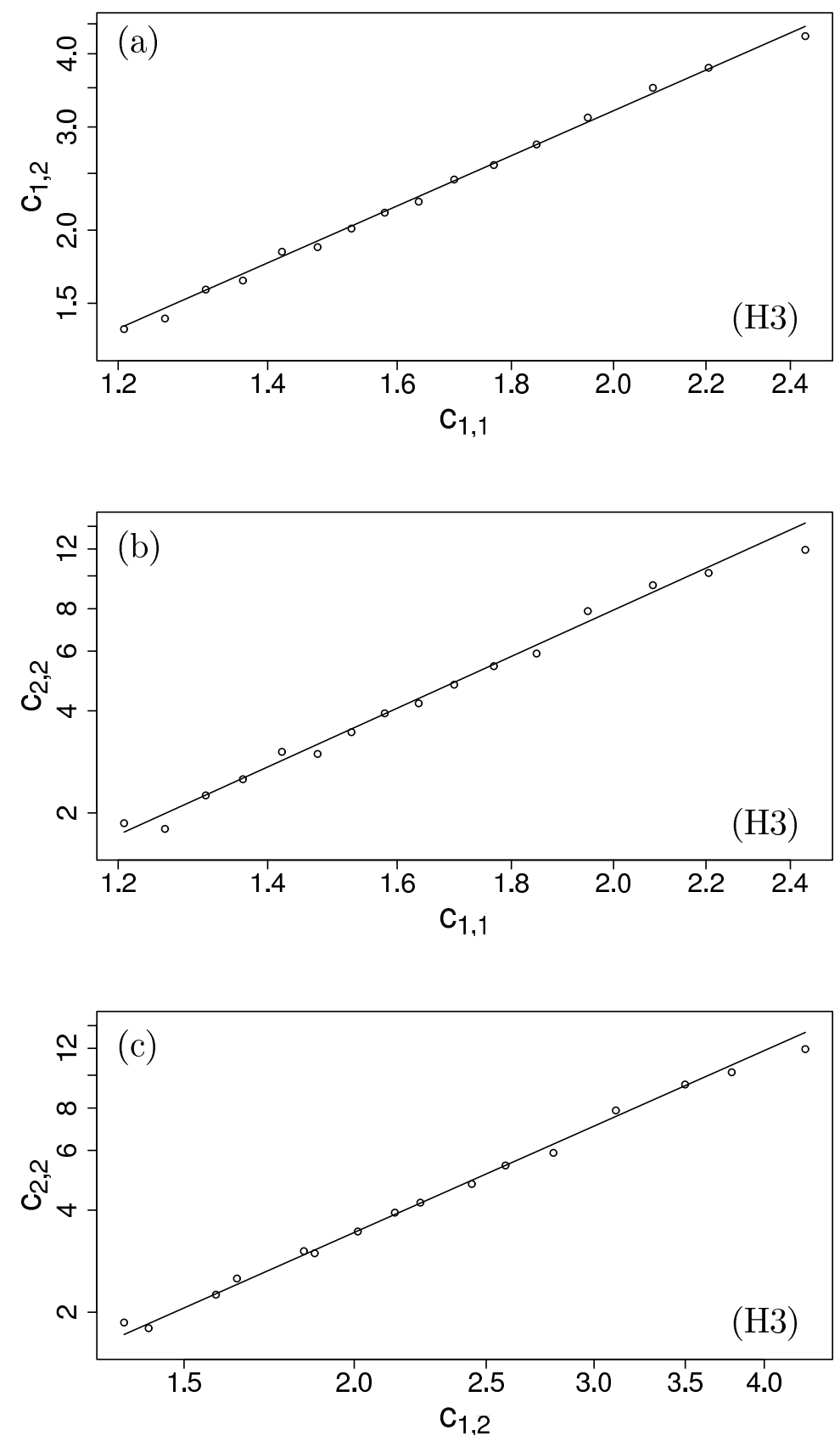

Figure 3: Energy dissipation correlators $c_{n_{1}, n_{2}}$ as a function of $c_{m_{1}, m_{2}}$ for data set (H3) and orders (a) $\left(n_{1}, n_{2}\right)=(1,2),\left(m_{1}, m_{2}\right)=(1,1)$, (b) $\left(n_{1}, n_{2}\right)=$ $(2,2),\left(m_{1}, m_{2}\right)=(1,1)$ and $(\mathrm{c})\left(n_{1}, n_{2}\right)=(2,2),\left(m_{1}, m_{2}\right)=(1,2)$, in double logarithmic representation. The straight line is of the form (a) $\left(c_{1,1}\right)^{1.68}$, (b) $\left(c_{1,1}\right)^{2.99}$ and $(\mathrm{c})\left(c_{1,2}\right)^{1.78}$. 

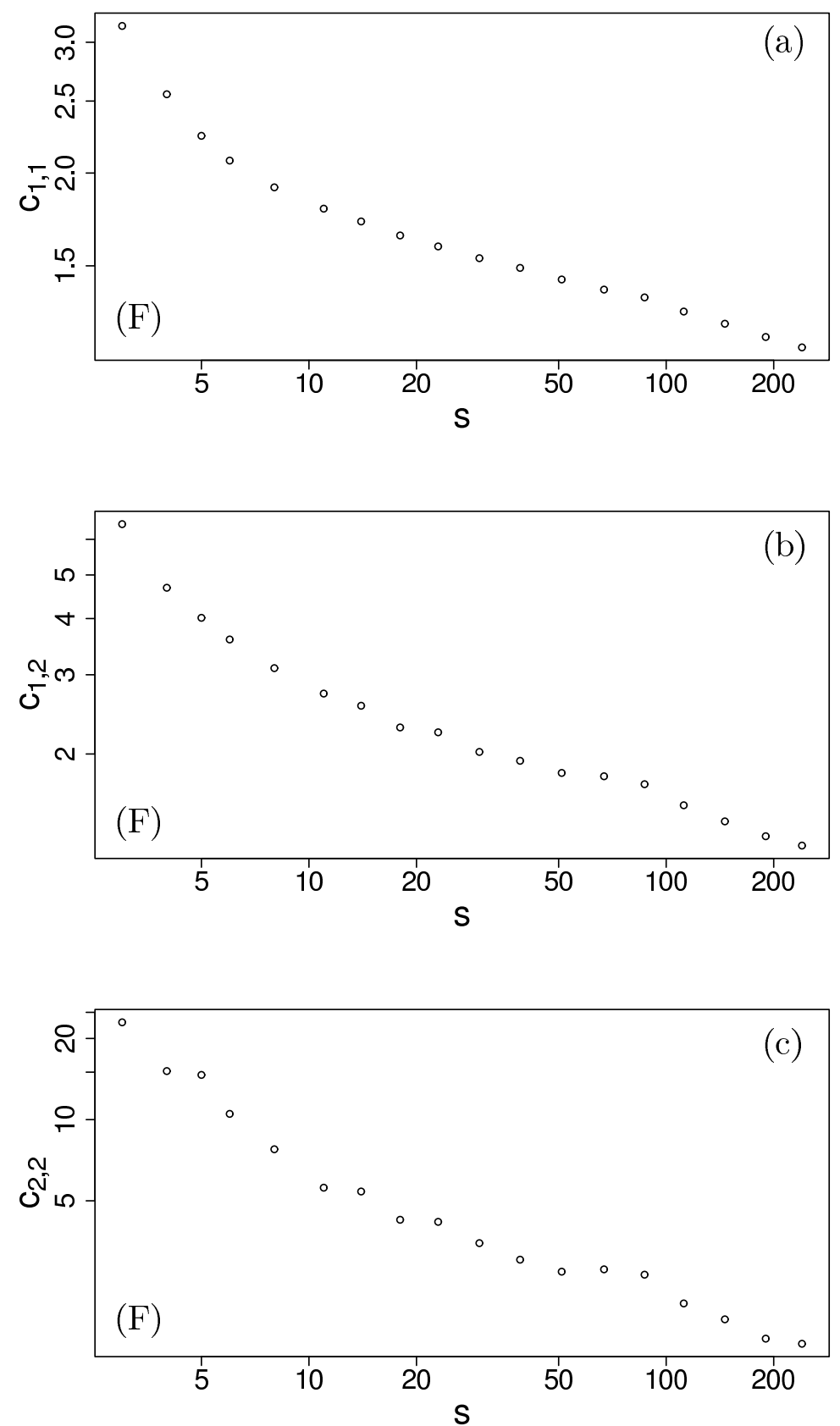

Figure 4: Energy dissipation correlators $c_{n_{1}, n_{2}}$ as a function of the lag $s$ (in units of the finest resolution) for data set (F) and orders (a) $\left(n_{1}, n_{2}\right)=$ $(1,1)$, (b) $\left(n_{1}, n_{2}\right)=(1,2)$ and $(\mathrm{c})\left(n_{1}, n_{2}\right)=(2,2)$ in double logarithmic representation. 

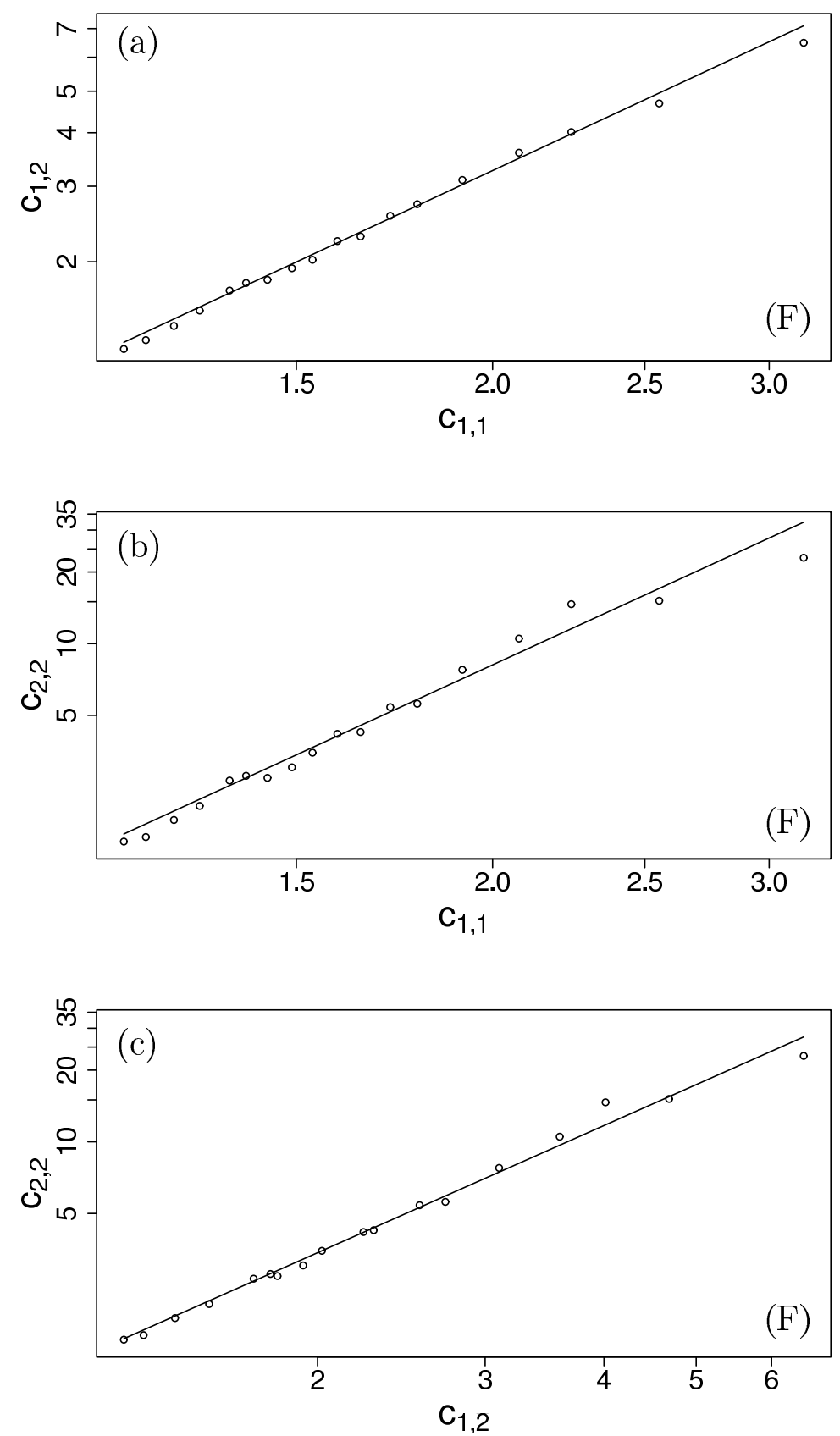

Figure 5: Energy dissipation correlators $c_{n_{1}, n_{2}}$ as a function of $c_{m_{1}, m_{2}}$ for data set $(\mathrm{F})$ and orders $(\mathrm{a})\left(n_{1}, n_{2}\right)=(1,2),\left(m_{1}, m_{2}\right)=(1,1)$, (b) $\left(n_{1}, n_{2}\right)=(2,2)$, $\left(m_{1}, m_{2}\right)=(1,1)$ and $(\mathrm{c})\left(n_{1}, n_{2}\right)=(2,2),\left(m_{1}, m_{2}\right)=(1,2)$, in double logarithmic representation. The straight line is of the form (a) $\left(c_{1,1}\right)^{1.71}$, (b) $\left(c_{1,1}\right)^{3.03}$ and $(\mathrm{c})\left(c_{1,2}\right)^{1.77}$. 

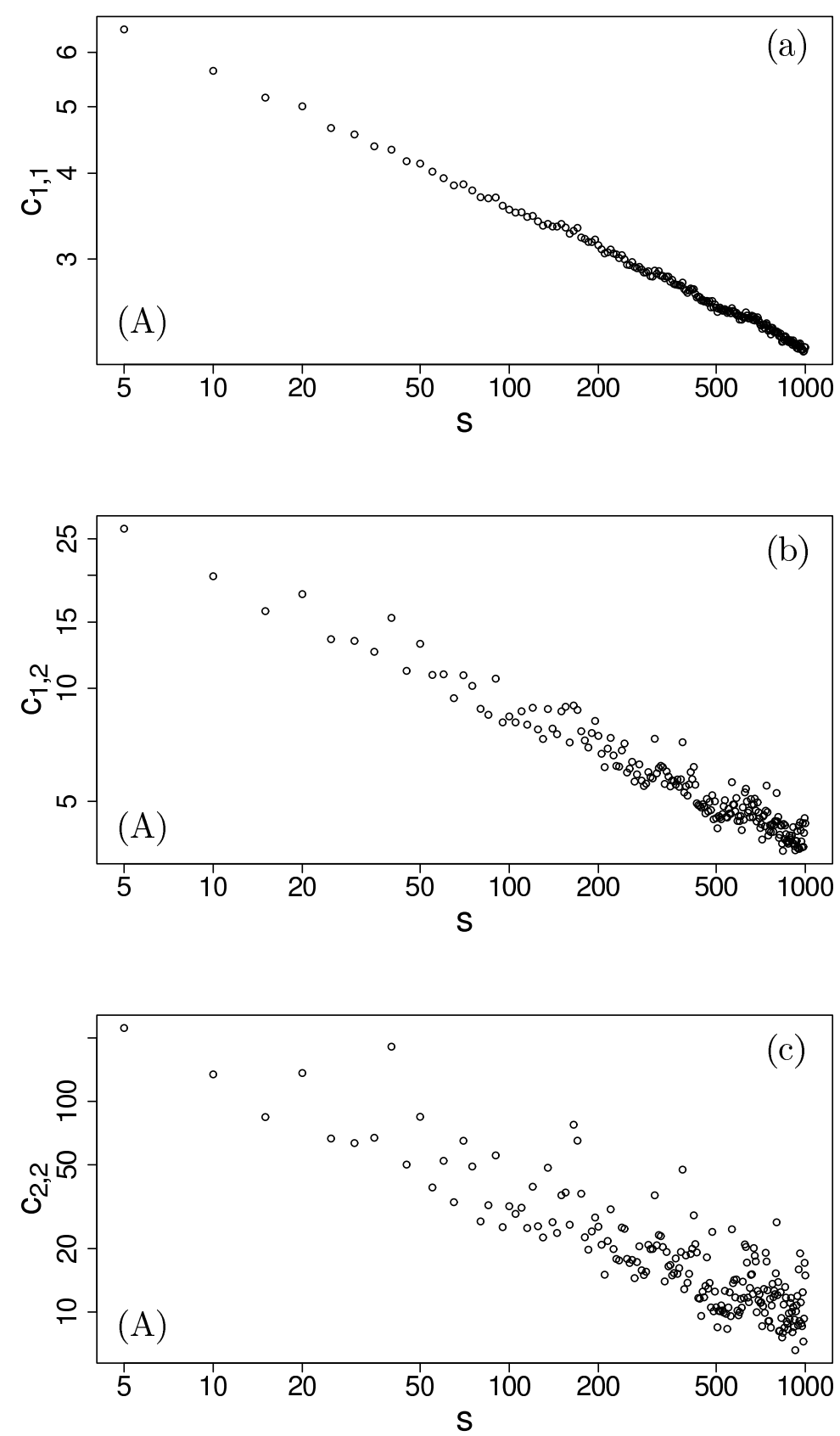

Figure 6: Energy dissipation correlators $c_{n_{1}, n_{2}}$ as a function of the lag $s$ (in units of the finest resolution) for data set (A) and orders (a) $\left(n_{1}, n_{2}\right)=$ $(1,1)$, (b) $\left(n_{1}, n_{2}\right)=(1,2)$ and $(\mathrm{c})\left(n_{1}, n_{2}\right)=(2,2)$ in double logarithmic representation. 

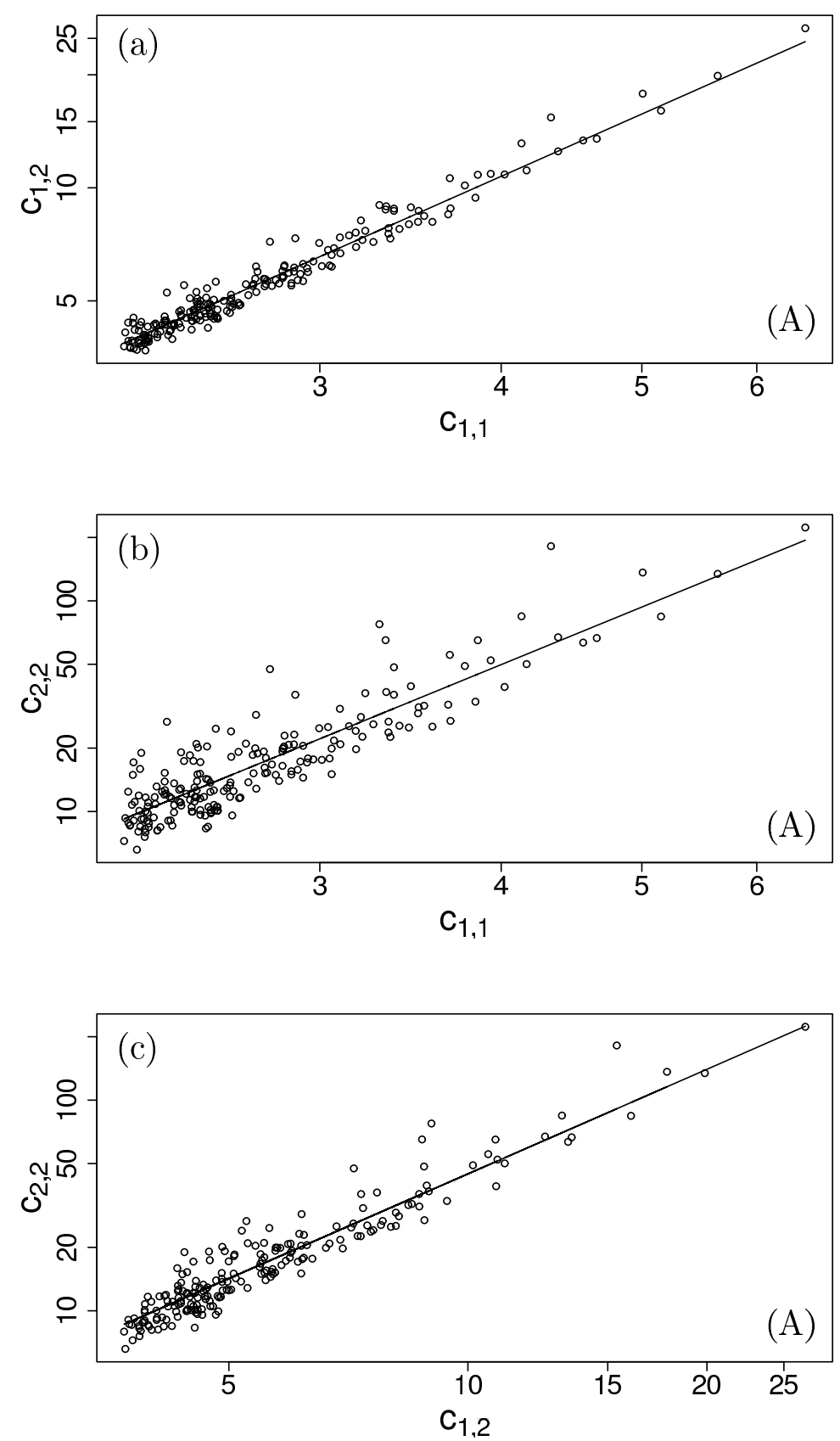

Figure 7: Energy dissipation correlators $c_{n_{1}, n_{2}}$ as a function of $c_{m_{1}, m_{2}}$ for data set (A) and orders (a) $\left(n_{1}, n_{2}\right)=(1,2),\left(m_{1}, m_{2}\right)=(1,1)$, (b) $\left(n_{1}, n_{2}\right)=$ $(2,2),\left(m_{1}, m_{2}\right)=(1,1)$ and $(\mathrm{c})\left(n_{1}, n_{2}\right)=(2,2),\left(m_{1}, m_{2}\right)=(1,2)$, in double logarithmic representation. The straight line is of the form (a) $\left(c_{1,1}\right)^{1.71}$, (b) $\left(c_{1,1}\right)^{2.82}$ and $(\mathrm{c})\left(c_{1,2}\right)^{1.65}$. 

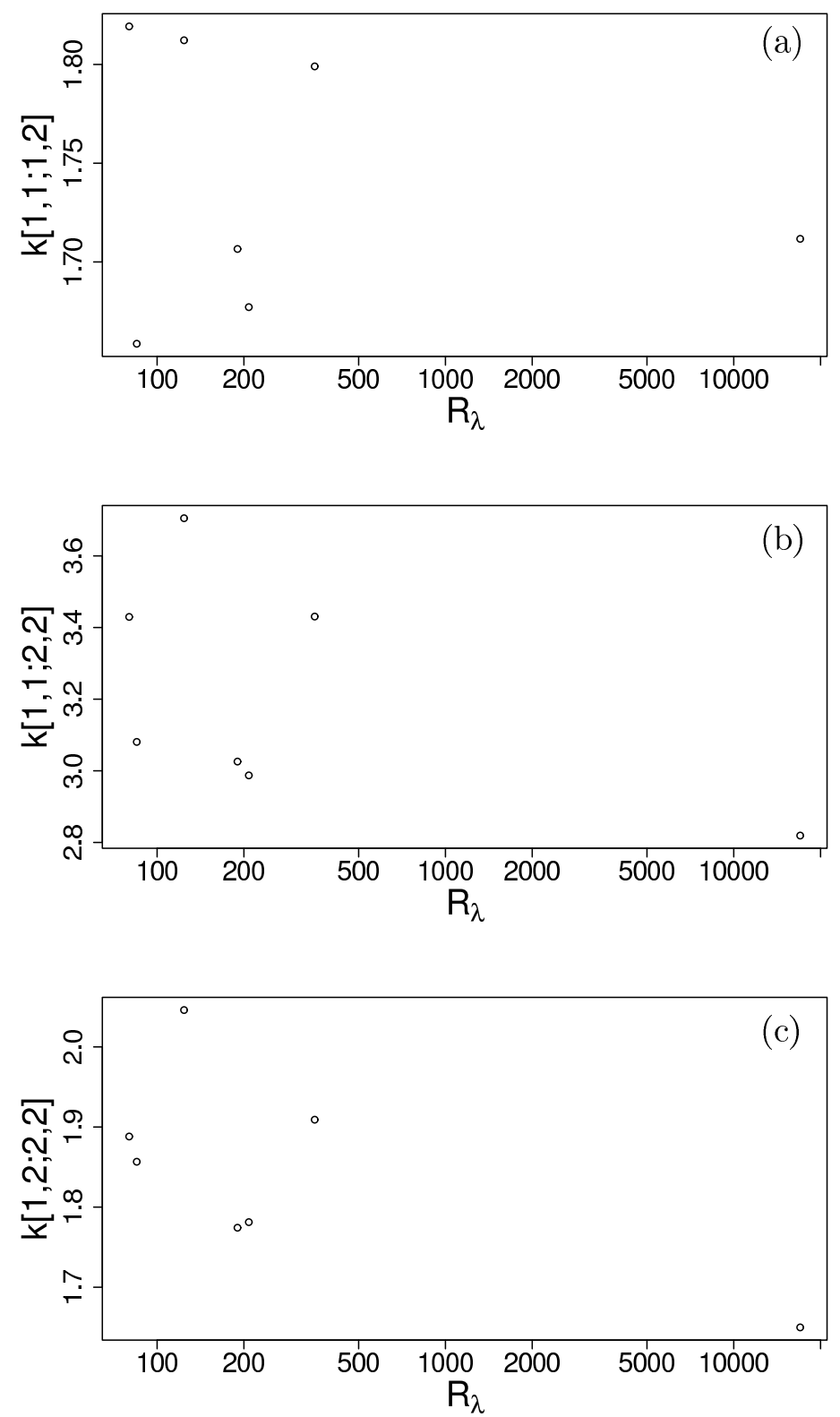

Figure 8: Self-scaling exponents $k\left[m_{1}, m_{2} ; n_{1}, n_{2}\right]$ of order (a) $\left(n_{1}, n_{2}\right)=$ $(1,2),\left(m_{1}, m_{2}\right)=(1,1),(\mathrm{b})\left(n_{1}, n_{2}\right)=(2,2),\left(m_{1}, m_{2}\right)=(1,1)$ and (c) $\left(n_{1}, n_{2}\right)=(2,2),\left(m_{1}, m_{2}\right)=(1,2)$ as a function of the Taylor Reynolds number $R_{\lambda}$. Abscissa is represented in logarithmic scales. 


\section{Discussion}

Our empirical findings clearly support the self-scaling relation (6) for not too small scales $s$. The deviations at very small scales can not be explained within the simple ansatz (3). To account for these, we propose, instead of $h\left(x-x^{\prime}, t-t\right) \equiv 1$, as in definition (3), to introduce a non-constant weightfunction $h$

$$
\epsilon(x, t)=\exp \left\{\int_{A_{t}(x)} h\left(x-x^{\prime}, t-t\right) Z\left(\mathrm{~d} x^{\prime} \times \mathrm{d} t^{\prime}\right)\right\} .
$$

This more general choice weights the contributions to $\epsilon(x, t)$ from the background field $Z$ differently. A natural choice would be of the form $h\left(x-x^{\prime}, t-t\right)=h\left(\left|x-x^{\prime}\right|,\left|t-t^{\prime}\right|\right)$ that measures the distance of the contributions of $Z$ at point $\left(x^{\prime}, t^{\prime}\right)$ to the field $\epsilon$ at point $(x, t)$ in a suitable norm. The spatio-temporal variation of this distance-function $h$ can not be analysed using a one-dimensional time series. Suggested by our empirical results, for low and moderate Reynolds numbers, we expect $h$ to vary slowly for small $\left|x-x^{\prime}\right|$ while being constant above some treshold $\left|x-x^{\prime}\right|=x_{0}$. Here $x_{0}$ is to be identified with scales where deviations from (6) occur. For $\left|x-x^{\prime}\right|>x_{0}$, one can always set $h\left(\left|x-x^{\prime}\right|\right) \equiv 1$.

The apparent Reynolds number independence of the self-scaling exponents $k\left[m_{1}, m_{2} ; n_{1}, n_{2}\right]$ and the strong Reynolds number dependence of the intermittency exponent $\mu$ [22] allows to draw some important conclusions about the dependence of the background field $Z$ on $R_{\lambda}$. The variation of $\mu$ with $R_{\lambda}$ indicates the non-universal character of the background field $Z$. In other words, the cumulant function $\mathrm{K}[n]=\mathrm{K}\left[n, R_{\lambda}\right]$ changes with the Reynolds number (and possibly with the type of experiment performed). However, the Reynolds number independence of $k\left[m_{1}, m_{2} ; n_{1}, n_{2}\right]$ implies

$$
\mathrm{K}\left[n, R_{\lambda}\right]=f_{n}\left(\mathrm{~K}\left[2, R_{\lambda}\right]\right),
$$

where the functions $f_{n}$ are universal (independent of $R_{\lambda}$ and the type of experiment) and only depend on the order $n$. (13) follows immediately by iterating (7) for $\left(n_{1}, n_{2}\right)=(1,1)$ and $\left(m_{1}, m_{2}\right)=(1, n-1)$ and using the normalisation $\mathrm{K}[1]=0$. For the universal functions $f_{n}$ we get

$$
f_{n}(x)=x\left(1+\sum_{i=2}^{n-1} k[1, i ; 1,1]^{-1}\right), n \geq 3 .
$$

Thus the background field $Z$ can be characterized by a single parameter $\mathrm{K}\left[2, R_{\lambda}\right]=\overline{\mathrm{K}}\left[2, R_{\lambda}\right]$ that contains all individual characteristics of the experimental situations. 
Furthermore, we conclude the identification

$$
\mathrm{K}\left[2, R_{\lambda}\right]=\mu
$$

designating the intermittency exponent $\mu$ as the only control parameter of the background field $Z$. To see this, we note that a proper definition of the intermittency exponent requires $c_{1,1}(s) \propto s^{-\mu}$ for a certain range of scales $s$. Comparing this scaling relation with $(5)$ for $\left(n_{1}, n_{2}\right)=(1,1)$ gives $a \overline{\mathrm{K}}\left[2, R_{\lambda}\right]=$ $\mu$, where $a$ is a positive constant. $a$ can simply be absorbed in the definition of the background field $Z$, establishing the identification (15).

\section{Conclusions}

The observed self-scaling of energy dissipation correlators clearly supports the multiplicative ansatz (3). Further motivation for this type of multiplicative structure is reported in [4], fitting two- and three point correlations of the high Reynolds number energy dissipation field with high accuracy, and in [2], establishing the connection to the multifractal picture of the energy dissipation. In these works, all calculations can be done analytically, a major advantage of the Lévy based modeling. The analytical tractability of the Lévy based model is highly advantageous for inference. The next step is a closer inference on the shape of the ambit set $A_{t}(x)$ and the distribution of $Z$ in their dependence on the Reynolds number. The latter determines the distribution of $\epsilon(x, t)$ since marginally $\epsilon(x, t)=\exp \left\{Z\left(A_{t}(x)\right)\right\}$.

\section{References}

[1] J. Schmiegel, PhD Thesis, TU Dresden (2002).

[2] O.E. Barndorff-Nielsen, H.C. Eggers, M. Greiner and J. Schmiegel, MAPHYSTO Research Report 2003-33 (http://www.maphysto.dk) (2003).

[3] O.E. Barndorff-Nielsen and J. Schmiegel, Uspekhi Mat. Nauk 159 (2004) 63.

[4] J. Schmiegel, J. Cleve, H.C. Eggers, B.R. Pearson and M. Greiner, Phys. Lett. A 320 (2004) 247.

[5] C. Meneveau and K.R. Sreenivasan, J. Fluid Mech. 224 (1991) 429.

[6] B. Jouault, P. Lipa and M. Greiner, Phys. Rev. E 59 (1999) 2451. 
[7] B. Jouault, M. Greiner and P. Lipa, Physica D 136 (2000) 125.

[8] J. Cleve and M. Greiner, Phys. Lett. A 273 (2000) 104.

[9] F.G. Schmitt, Eur. Phys. J. B 34 (2003) 85.

[10] J.F. Muzy and E. Bacry, Phys. Rev. E 66, (2002) 056121.

[11] R. Benzi, S. Ciliberto, R. Tripiccione, C. Baudet, F. Massaioli and S. Succi, Phys. Rev. E 48, (1993) 29.

[12] O. Kallenberg, Random Measures, (Akademie Verlag, Berlin, 1989).

[13] S. Kwapien and W.A. Woyczynski, Random Series and Stochastic Integrals: Single and Multiple, (Birkhäuser, Basel, 1992).

[14] In this context, continuity refers to the definition of the observable $\epsilon(x, t)$ for a continuous range of points $(x, t)$.

[15] B. Dhruva, PhD Thesis, Yale University (2000).

[16] K.R. Sreenivasan and B. Dhruva, Prog. Theor. Phys. Suppl. 130, (1998) 103.

[17] O. Chanal, B. Chebaud, B. Castaing and B. Hébral, Eur. Phys. J. B 17, (2000) 309.

[18] C. Renner, J. Peinke and R. Friedrich, J. Fluid Mech. 433, 383-409 (2001).

[19] R.A. Antonia and B.R. Pearson, Phys. Rev. E 62, 8086-8090 (2000).

[20] J. Cleve, M. Greiner and K.R. Sreenivasan, Europhys. Lett. 61, (2003) 756 .

[21] K.R. Sreenivasan and P. Kailasnath, Phys. Fluids 5, (1992) 512.

[22] J. Cleve, M. Greiner, B.R. Pearson and K.R. Sreenivasan, Phys. Rev. E 69, (2004) 066316.

[23] R.A. Antonia and B.R. Satyaprakash, J. Fluid Mech. 119, (1982) 55. 